\title{
EXIGÊNCIA AGROCLIMÁTICA DA CULTURA DO MILHO (Zea mays)
}

\author{
Luciano Junior Maldaner ${ }^{2}$, Kelly Horing ${ }^{3}$, Jaciara Fernanda Schneider ${ }^{4}$, Jianice Pires Frigo ${ }^{5}$, \\ Késia Damaris de Azevedo ${ }^{6}$, Anderson Eduardo Grzesiuck $^{7}$
}

\author{
${ }^{1}$ Aceito para publicação no $1^{\circ}$ trimestre de 2014 \\ ${ }^{2}$ Acadêmico do curso de Agronomia pela Universidade Federal do Paraná UFPR - Setor Palotina, Brasil, \\ luciano_maldaner@hotmail.com; \\ ${ }^{3}$ Acadêmico do curso de Agronomia pela Universidade Federal do Paraná UFPR - Setor Palotina, Brasil, \\ kellyhoring@gmail.com; \\ ${ }^{4}$ Acadêmico do curso de Agronomia pela Universidade Federal do Paraná UFPR - Setor Palotina, Brasil, \\ jaciarafernandaschneider@hotmail.com; \\ ${ }^{5}$ Graduanda em agronomia na Universidade Regional Integrada do Alto Uruguai e das Missões - URI Campus de \\ Santiago-RS, Brasil, jifrigo@yahoo.com; \\ ${ }^{6}$ Graduada em Tecnologia em Biotecnologia na Universidade Federal do Paraná, Palotina - PR, Brasil, \\ kesia.damaris@gmail.com; \\ ${ }^{7}$ Acadêmico do curso de Agronomia pela Universidade Federal do Paraná UFPR - Setor Palotina, Brasil, \\ anderson.grzesiuck@gmail.com.
}

\section{Resumo}

A cultura do milho faz parte das culturas energéticas mais produzidas no Brasil, um país altamente competitivo na produção e em sua eficácia, favorecidos pelas condições agroclimáticas e técnicas de cultivo, já especializadas e com o estudo das interferências nesta produção. O clima pode ser considerado o fator de maior risco para a atividade agrícola, que contribui definitivamente para o sucesso da cultura do milho ou de qualquer outra. Para alcançar as altas produções que a cultura de milho tem atingido, é adotado a técnica do zoneamento agrícola de risco climático, que atende 24 estados e 40 culturas, além de contemplar a monocultura do milho, e atendendo o consórcio de milho e braquiária. Os principais fatores climáticos que podem afetar a planta são; a radiação solar, temperatura e precipitação, após identificar estes dá-se início ao zoneamento agrícola da cultura, com objetivo de identificar as regiões e épocas aptas ao cultivo. Para criar um zoneamento da cultura deve-se avaliar todos os parâmetros climáticos que afetam as plantas, como; coeficiente de cultura, evapotranspiração potencial, precipitação e temperatura, ciclo e fase fenológica da cultura e disponibilidade máxima de água no solo. 
Palavra-chave: zoneamento climático, fatores climáticos, efeitos fisiológicos.

\title{
AGROCLIMATIC REQUIREMENT OF MAIZE
}

\begin{abstract}
The corn crop is part of energy crops more produced in Brazil, a country highly competitive in production and in its effectiveness, favored by agro-climatic conditions and cultivation techniques, already specialized and with the study of interference in this production .The climate can be considered to be the factor that has the greatest risk for agricultural activity, that contribute to the success of the culture of corn or any other. To meet this specialization that the maize crop has reached, develops the technique of agricultural zoning of climatic risk, given 24 states and 40 cultures, and contemplate the monoculture of corn, meets the consortium of corn and brachialis. After being characterized the main climatic factors that may affect the plant, it is beginning to agricultural zoning of the plant, with the objective to identify the regions and times suitable for cultivation. To create a zoning of a culture must evaluate all parameters that affect with influence the plant in all methods; amount of rainfall and temperature, potential evapotranspiration, cycle and phonological stage of culture, crop coefficient and maximum availability of water in the soil.
\end{abstract}

Keyword: climate zones, climatic factors, physiological effects.

\section{Introdução}

Para a $1^{\text {a }}$ safra de 2014 o Brasil irá cultivar 6.435,4 mil hectares, produzindo 31.423,5 mil toneladas de milho, tendo o Paraná como o principal produtor com 670,0 mil hectares e 5.508,7 mil toneladas de milho, porém com uma redução de $23,7 \%$ na área plantada em função dos baixos preços, sério ataque de Spodoptera frugiperda na fase vegetativa e a instabilidade do clima (COMPANIA NACIONA DE ABASTECIMENTO - CONAB, 2014).

O sucesso da produção está ligado ao planejamento da atividade, quanto mais eficiente for o planejamento menor será os fatores de risco. O milho pode ter sua produção afetada por diversos fatores como, eficiência metabólica, interceptação da radiação por Dossel, capacidade de dreno e a eficiência da translocação de foto-assimilados, onde o conjunto destes fatores associados acarretam sérios danos a cultura (CORRÊA et al., 2009). 
Na agricultura, a redução de custo e do insucesso são fatores indispensáveis para a competividade, porém é difícil prever exatamente o clima devido sua variação ao longo do tempo e do espaço. O clima pode ser considerado o fator de maior risco para a atividade agrícola, sendo a água, e ou, a chuva um dos elementos indispensáveis que contribuem definitivamente para o sucesso da cultura (SILVA; et al., 2004).

Para se obter o máximo do potencial da cultura o Ministério da Agricultura desenvolve desde 1996 a técnica do zoneamento agrícola de risco climático, atendendo 24 estados e 40 culturas, além de abranger o cultivo do milho, o zoneamento atende o consórcio de milho e braquiária (MINISTÉRIO DA AGRICULTURA, PECUÁRIA E ABASTECIMENTO MAPA, 2014).

Graças a uma redução na área de cultivo do milho, este trabalho busca incentivar o cultivo do milho através da adoção do planejamento do cultivo, levando em conta os principais fatores climáticos, que acabam reduzindo a produção, e associados a diversos fatores tem se tornado uma das razões para redução da área de cultivo.

Devido à grande importância econômica da cultura do milho para o Paraná, está revisão literária tem por objetivo associar os efeitos agroclimáticos na distribuição da cultura pelo pais, relacionando as técnicas utilizadas para o zoneamento.

\section{Influência Agroclimáticas no Milho}

De acordo com Majerowicz (2004), o milho possui metabolismo $\mathrm{C}_{4}$, que lhe confere uma taxa fotossintética líquida maior ao ser comparado a $\mathrm{C}_{3}$, pois apresenta baixa perda de água podendo ser cultivado em ambientes quentes com alta intensidade luminosa, se adaptando bem ao clima tropical pela sua baixa fotorrespiração (LACERDA, 2007).

A cultura do milho é afetada por fatores climáticos, o autor Durães (2006) afirma que estes fatores são: “[...] primários; latitude, altitude, chuva, topografia, textura do solo, composição do solo; e fatores climáticos secundários; radiação solar, comprimento do dia, temperatura, água no solo, aeração do solo, minerais do solo [...]”. A interação negativa destes fatores ocasiona a redução na produção (DURÃES, 2006). Entre os diversos fatores se destacam a radiação solar, temperatura e precipitação, que reduzem o crescimento e o desenvolvimento da planta, sendo que sua ação varia com o estádio fenológico da planta, assim cada estádio possui níveis toleráveis diferentes (ALVES et al., 2010).

\section{Radiação Solar}

Revista Brasileira de Energias Renováveis, v. 3, p. 13-23, 2014 
A radiação solar está totalmente ligada a produção direta de massa no milho, portanto a relação da massa de matéria seca acumulada e a radiação interceptada pelo vegetal num período de tempo, obtém-se a eficiência na produção de massa da planta (ROMANO, 2005).

Para a planta realizar a fotossíntese bioquímica é necessário energia na forma de ATP e NADPH, essa energia é proveniente dos cloroplastos, que usam a energia luminosa na forma de radiação solar e a convertem em energia química (ATP e NADPH), sendo está energia fundamental para os processos fisiológicos da planta (TAIZ et al., 2009). A produção do milho está totalmente relacionada a radiação, pois cerca de 78,5\% da produtividade é dependente da radiação luminosa (ROMANO, 2005).

Segundo Bevilacqua (2012), a radiação solar é essêncial para o desenvolvimento do milho, pois sem este o processo fotossintético é inibido e consequentemente ocorre redução na produção, isto porque a fixação de $\mathrm{CO}_{2}$ fornece cerca de $90 \%$ da matéria seca, e uma redução de 30 a $40 \%$ da radiação por um logo período pode acarretar na redução da produção e atraso na maturação. O máximo aproveitamento da radiação ocorre no pré-florescimento e no enchimento de grãos, sendo está fase o período mais crítico (RODRIGUES et al., 2011).

Grossi et al. (2011), realizou um estudo sobre a influência da radiação solar no período de 1987 a 2006, através do coeficiente de regressão constatou que a radiação solar pode ocasionar redução na produção do milho, o coeficiente de regressão teve uma significância de $10 \%$ em relação ao período antes do enchimento dos grãos, indicando a influência da radiação solar sobre a produção final. A redução de 16,85\% da radiação solar de 1989 para 1992 ocasionou uma perca de $43,21 \%$ na produção, porém esta diminuição teve influência das baixas temperaturas, pois os anos que apresentaram as menores produtividades foram os que possuíam menor incidência solar e temperaturas mais baixas.

Segundo Romano (2005), a arquitetura de Dossel é um dos fatores que mais afetam a interceptação da luz, onde as maiores produtividades são observadas em plantas com arquitetura ereta, onde os raios solares possuem uma maior área para incidir, já as plantas com folhas mais pendidas possuem taxas assimilatórias menores e consequentemente menor produtividade de grãos.

\section{Temperatura}

De acordo com Rodrigues et al. (2011), caso o solo tenha umidade suficiente o milho se desenvolve bem em altas temperaturas. Cruz et al. (2010) destaca que o milho suporta temperaturas noturnas de até $30^{\circ} \mathrm{C}$ por longos períodos. Grossi et al. (2011), também concluiu 
que temperaturas noturnas elevadas reduzem a produção, pois a noite a planta continua respirando, mas paralisa a fotossíntese, ocorrendo o consumo de produtos metabólicos produzidos de dia. Em temperaturas menores de $10{ }^{\circ} \mathrm{C}$ o desenvolvimento é quase nulo, com tudo, a temperatura deve oscilar de 10 a $30^{\circ} \mathrm{C}$.

Segundo Taiz et al.(2009), a planta do milho possui metabolismo C4, isto é, a planta possui a enzima PEPCase (fosfoenolpiruvatocaborxilase) que apresenta afinidade apenas com o $\mathrm{CO}_{2}$, assim não perde energia com a fotorrespiração. A PEPCase e PEP (fosfoenolpiruvato) são as principais responsáveis por manter a concentração elevada de $\mathrm{CO}_{2}$ no sítio ativo da RUBISCO (ribose-1,5-bifosfato carboxilase/oxidade), com isso o ciclo de Calvin sempre possuirá $\mathrm{CO}_{2}$, assim poderá manter seus estômatos fechados por um período maior e suportar temperaturas mais altas e níveis elevados de radiação. Isso garante que o milho sintetize fotoassimilados com baixa quantidade de água e temperaturas mais elevadas.

A temperatura para germinação até a maturação deve ficar próxima dos $25^{\circ} \mathrm{C}$ (EMBRAPA, 2004). Ao comparar temperaturas médias de $25^{\circ} \mathrm{C}, 21^{\circ} \mathrm{C}$ e $18^{\circ} \mathrm{C}$, foi observado um melhor desempenho na média de $21^{\circ} \mathrm{C}$, sendo que apresentou maior produção de grãos e matéria seca, isso pode ser explicado pelo fato que o milho necessita acumular determinadas quantidades de unidades calóricas em cada estádio e caso ocorra elevadas temperaturas o ciclo da planta é acelerado devido ao acumulo de graus dias (GD), isso gera consequências como a redução da produção (CRUZ et al., 2010).

Grossi et al. (2011) observou que temperaturas máximas não influenciam a produtividade do milho. As regiões aptas para o cultivo do milho devem possuir temperaturas superiores a $15^{\circ} \mathrm{C}$ e não ter ocorrência de geadas durante todo o ciclo do milho (ALVES et al., 2010).

Segundo Schons et al. (2009), o milho possui uma constante térmica de aproximadamente $900{ }^{\circ} \mathrm{Cd}(\mathrm{CT})$, de acordo com a temperatura acumulada necessária para atingira a polinização, os GD são divididos em três grupos; assim as cultivares que necessitam de 780 a 830 GD são designadas cultivares supre-precoces, com 831 a 890 GD precoces e 891 a 1.200 GD cultivares tardias (FANCELLI et al., 2000).

De acordo com Fiorini et al. (2012), na segunda quinzena de novembro e dezembro os híbridos de milho não são afetados pelos GD, porém os híbridos cultivados em dezembro o florescimento e os GD são maiores. O autor ainda destaca que foi realizado poucos experimentos, assim não se pode ter conclusões exatas sobre o desempenho dos híbridos em relação a influência dos graus dias, sendo que este parâmetro deve ser analisado em grandes 
áreas nos mais diversos pontos do país, como é feito pelas empresas de sementes, gerando resultados mais confiáveis.

\section{Precipitação}

Segundo a Embrapa (2004), o milho necessita de no mínimo 350 a 500 mm de chuva por ciclo, pluviometria menores irá exigir o uso de irrigação, sendo que a precipitação ideal gira em torno de 500 a 800 mm Porém, de acordo com Bergamaschi et al (2006), a necessidade hídrica do milho varia de 200 a 400mm para o ciclo completo, mas estes valores variam com os diferentes locais e épocas de plantio.

Os fenômenos El Niño e La Niña afetam a distribuição de chuvas no sul do Brasil, onde o El Niño causa aumento das temperaturas médias e da pluviometria, já o La Niña causa longos períodos de seca (FONSECA, 2010).

Devido à grande influência deste fenômeno Bergamaschi et al. (2004), analisou a produção do milho no Rio Grande do Sul sob o efeito dos dois fenômenos, o autor observou que a média do estado foi 2.422 em condição de La Niña e 3.750 em condição de El Niña.

Segundo Bergamaschi et al. (2004), a produtividade é muito variável sob ação dos fenômenos La Niña e El Niño, pois mesmo que o milho esteja em um período de seca, mas receba a quantidade correta de chuva nos períodos de desenvolvimento critico (emissão florescência masculina até enchimento do grão), a planta irá ter uma boa produção. O mesmo autor observou produção $8000 \mathrm{Kg} / \mathrm{ha}$ em condição de La Niña e $2000 \mathrm{Kg} / \mathrm{ha}$ em condição de El Niño, porém plantas com maior produção não tiveram déficit hídrico no período crítico de produção.

Segundo Bergamaschi et al. (2004; 2006), a irrigação pode ser uma das melhores soluções para aumentar a produção de plantas que são afetadas pelo estresse hídrico, onde se pode ter um acréscimo de até $70 \%$ na produção com o uso da irrigação, onde a redução de $44 \%$ do total da água pode acarretar em uma perca 12\% na produção total de grãos. Wagner (2009), também observou uma diminuição de $50 \%$ na produção em comparação a plantas irrigadas, no município de Guarapuava sob influência de déficit hídrico.

A quantidade de água irrigada não tem grande influência na produção, mas o período em que ocorre a irrigado é o que vai influenciar, onde 60 a $80 \%$ da pluviometria foram suficientes para altas produções de grãos de milho, mesmo que a Capacidade de Água Disponível (CAD) esteja próximo do ponto de murcha permanente (PMP), os grãos por espiga 
e o número de espigas por planta são menos afetados caso não ocorra estresse hídrico no período crítico (BERGAMASCHI et al., 2004).

Wagner (2009), realizou experimentos em Guarapuava e constatou que a produção do milho independe da época de semeadura, pois a probabilidade de perdas por déficit hídrico é maior que a não ocorrência, mas plantas semeadas no período recomendado pelo zoneamento terão uma maior probabilidade de terem sucesso.

O déficit hídrico acaba limitando a fotossíntese no cloroplasto, pois é necessário a quebra da molécula de água para repor os elétrons perdidos pela clorofila para fazer a redução do NADPH. A água é responsável pelo transporte de nutrientes, caso o solo esteja no PMP, não ocorrerá a absorção de nutrientes por fluxo de massa, isto é, pela diferença de potencial hídrico (KERBAUY, 2004).

O déficit de água no solo é refletido para a planta, assim os estômatos permanecem fechados para evitar a transpiração, consequentemente não ocorrerá o transporte de fotoassimilados, aumentando a concentração destes na fonte de produção, que refletira na redução da fotossíntese, pela alta concentração nas células companheiras (TAIZ et al., 2009).

\section{Zoneamento Agroclimático}

Caso as condições adversas de radiação solar, temperatura e precipitação permanecerem por períodos mais prolongados a planta vai desenvolver meios para se adaptar ao estresse, porém se a ação de uma ou o grupo de intemperes permanecer em condições extremas, a planta irá diminuir o seu metabolismo e a divisão celular, aumentado a produção de etileno e iniciando o processo de senescência (KERBAUY, 2004).

Segundo Coelho et al. (2004), a época de plantio do milho não afeta os custos de produção, porém afetara a produção final. O mesmo auto ressalta que cultivares semeados em épocas inadequadas podem perder até $30 \mathrm{Kg} / \mathrm{ha}$ por dia, e é devido a essas percas que é desenvolvido o zoneamento agrícola, para que a probabilidade de ocorrência de danos a cultura seja reduzido.

Após identificar como os principais fatores climáticos podem afetar a planta, dá-se início ao zoneamento agrícola, com o objetivo de identificar as regiões aptas ao cultivo. Para criar o zoneamento de uma cultura o MAPA avalia os seguintes parâmetros; coeficiente de cultura, evapotranspiração potencial, precipitação e temperatura, ciclo e fase fenológica da cultura, e a disponibilidade máxima de água no solo (BRASIL, 2013). 
Segundo o BRASIL (2013), o zoneamento agroclimático é a classificação de regiões onde é determinado a época de semeadura do milho. Para o estado do Paraná o MAPA estabeleceu os dias de 21/08 a 31/12 o período de plantio para o milho, sendo estas datas referentes a $1^{a}$ safra, também conhecida como safra de verão, onde são obtidas as melhores produtividades. A $2^{\circ}$ safra do milho é semeada nos dias $1 / 1$ a 28/02, também conhecido como safrinha, porém possui uma produtividade menor ao ser comparada com a safra de verão, devido a ação negativa do clima.

\section{Considerações Finais}

Concluiu-se que os efeitos climáticos vão definir o sucesso da cultura, pois os principais fatores que afetam a cultura é a radiação solar, precipitação e temperatura, que pode afetar o milho por ação conjunta ou individual, tendo as maiores perdas quando estes fatores agem no período crítico de desenvolvimento. Porém existe diversas técnicas para minimizar a probabilidade de queda da produção, sendo o zoneamento agrícola uma das mais importantes, pois leva em conta a região e a época de plantio mais apta para o cultivo. 


\section{Referências}

ALVES, F. Q. G.; SILVA, F. C.; COSTA, I. J. S.; DAVID, A. M. S. S.; SIMÕES, D. A. Qualidade fisiológica de híbridos de milho submetidos a diferentes temperaturas. Trabalho apresentado no 28. Congresso Nacional de Milho e Sorgo, Goiânia: Associação Brasileira de Milho e Sorgo, 2010.

BERGAMASCHI, H.; DALMAGO, G. A.; BERGONCI, J. I.; BIANCHI, C. A. M.; MULLER, A. G.; COMIRAN, F.; HECKLER, B. N. N. Distribuição hídrica no período crítico do milho e produção de grãos. Pesquisa Agropecuária Brasileira, Brasília, v.39, n.9, p.831-839, set. 2004.

BERGAMASCHI, H.; DALMAGO, G. A.; COMIRAN, F. et al. Déficit hídrico e produtividade na cultura do milho. Brasília, Pesquisa Agropecuária Brasileira, v. 41, n.2, p.243-249. 2006.

BEVILACQUA, L. B. Sazonalidade da concentração de $\mathrm{CO}_{2}$ atmosférico em um área agrícola no RS. 52 f. Dissertação (mestrado) - Universidade Federal de Santa Maria, Centro de Ciências Naturais e Exatas, Programa de Pós-Graduação em Física, RS, 2012.

BRASIL. Ministério da Agricultura, Pecuária e Abastecimento. Secretaria de Política Agrícola. Portaria n. 180, de 12 de novembro de 2013. São Paulo-SP, p. 33.

COELHO, A. M.; CRUZ, J. C.; FILHO, I. A. P. Desafios para obtenção de altas produtividades de milho. Sete Lagoas, MG, Embrapa Milho e Sorgo, 2004. 23p. (Embrapa Milho e Sorgo. Comunicado Técnico, 112).

CONAB. Acompanhamento da safra brasileira de grãos. v. 1 - Safra 2013/14, n. 6 - Sexto Levantamento, Brasília, p. 1-83, mar. 2014

CORRÊA, T. B. S.; FIGUEIREDO, A. F.; ALVES, A. C.; SOUZA, G. V. P.; CONCEIÇÃO, A. L. M.; FONTES, R. T. Zoneamento de riscos climáticos do consórcio feijão-milho no estado do Rio de Janeiro. Trabalho apresentado no 16. Congresso Brasileiro de Agrometeorologia, Belo Horizonte - MG, 2009.

CRUZ, J. C.; MElhoranÇA, A. L.; COElHO, A. M. et al. Cultivo do milho, 2010. Disponível em: <http://www.cnpms.embrapa.br/publicacoes/milho_6_ed/manejomilho.htm>. Acessado em: 25 de ABR. de 2014. 
DURÃES, F.O.M. Limitações fisiológicas do milho nas condições de plantio nas regiões tropicais baixas,2006. Artigo em Hypertext. Disponível em: <http://www.infobibos.com/Artigos/2007_1/limitemilho/index.htm>. Acesso em:27 de ABR. de 2014.

EMBRAPA. Manual de segurança e qualidade para a cultura do milho. Brasília: Embrapa/Sede, 2004. 78 p.

FANCELLI, A.L.; DOURADO NETO, D. Produção de milho. Guaíba: Agropecuária, 2000. $360 \mathrm{p}$.

FIORINI, I. V. A.; FIORINI, F. V. A.; PINHO, R. G. V.; LASMAR, C. S.; CARVALHO, M. L. Avaliação do Ciclo, Somatório de Graus Dias e Produtividade de Híbridos de Milho. Trabalho apresentado no 29.Congresso Nacional de Milho e Sorgo. Águas de Lindóia-SP, 2012.

FONSECA, M. Conheça os fenómenos El Niño e La Niña, 2010. Disponível em: <http://professormarcelus.blogspot.com.br/2010/07/conheca-os-fenomenos-el-nino-e-lanina.html>. Acessado em: 18 de MAI. De 2014.

GROSSI, M. C.; SILVA, R. F.; ANDRADE, C. L. T.; JUSTINO, F. Influência da radiação solar e da temperatura do ar na produtividade potencial simulada do milho (Zea mays) em Sete Lagoas, MG. Trabalho apresentado no 17. Congresso Brasileiro de Agrometeorologia. Guarapari-ES, 2011.

KERBAUY, G. B. Fisiologia Vegetal. 1. ed. São Paulo: Guanabara Koogan S. A., 2004.

LACERDA, C. F. et al. Fisiologia vegetal. Fortaleza - Ce, 2007. (Apostila).

MAJEROWICZ, N. Fotossíntese. In: KERBAUY, G. B. Fisiologia vegetal. 1.ed. São Paulo: Guanabara Koogan, 2004. p. 114-178.

MAPA-Ministério da Agricultura Pecuária e Abastecimento. Zoneamento agrícola de risco climático, 2014. Disponível em: <http://www.agricultura.gov.br/politicaagricola/zoneamento-agricola>. Acessado em: 25 de ABR, de 2014. 
RODRIGUES, L. R.; SILVA, P. R. F.; FERREIRA, P. R. et al. Indicações técnicas para o cultivo do milho e do sorgo no Rio Grande do Sul: Safras 2011/2012 e 2012/2013. 1.ed. Porto Alegre: Fepagro, 2011. 140 p.

ROMANO, M. R. Desempenho fisiológico da cultura de milho com plantas de arquitetura contrastante: parâmetros para modelos de crescimento. Piracicaba, 2005. 100 p. Tese (Doutorado) - Escola Superior de Agricultura Luiz de Queiroz, 2005.

SCHONS, A.; STRECK, N. A.; STORCK, L. et al. Arranjos de plantas de mandioca e milho em cultivo solteiro e consorciado: crescimento, desenvolvimento e produtividade. Bragantia, Campinas, v.68, n.1, p.155-167, 2009.

SILVA, S. C.; SOARES, E. G. S. Zoneamento agroclimático, 2004. Disponível em: <http://www.agencia.cnptia.embrapa.br/gestor/feijao/arvore/CONTAG01_49_131120021510 3.html>. Acessado em: 25 de ABR. de 2014.

TAIZ, L. \& ZEIGER, E. Fisiologia Vegetal.4.ed. Porto Alegre: Artmed, 2009.

WAGNER, M. V. Características hidro-climáticas para a cultura do milho em Guarapuava-PR. Guarapuava, 2009. 91 p. Dissertação (Mestrado) - Universidade Estadual do Centro-Oeste, Programa de Pós-Graduação em Agronomia, área de concentração em Produção Vegetal, 2009. 